“( 2014 IEEE. Personal use of this material is permitted. Permission from IEEE must be obtained for all other uses, in any current or future media, including

reprinting/republishing this material for advertising or promotional purposes, creating new collective works, for resale or redistribution to servers or lists, or reuse of any copyrighted component of this work in other works." 


\title{
Users' Voice and Service Selection: An Empirical Study
}

\author{
Muneera Bano, Didar Zowghi \\ Faculty of Engineering and IT \\ University of Technology, Sydney \\ Sydney, Australia \\ Muneera.Bano@student.uts.edu.au,Didar.Zowghi@uts.edu.au
}

\begin{abstract}
Service Oriented software development saves time by reusing existing services and integrates them to create a new system. But selecting a service that satisfies the requirements of all concerned stakeholders is a challenging task. The situation has been exacerbated within the past few years with huge number of services available that offer similar functionalities where the analysts require additional information for making better decision for service selection. User feedback analysis has recently gained a lot of attention for its potential benefits in various areas of requirements engineering. The aim of this research is to evaluate the impact of feedback provided by the end users of the services, on the decision making process for the service selection. In this paper we present an empirical study that utilizes user feedback analysis for selection of a service among 92 available services with similar functionalities. The results show that in scenarios with significant number of services, it is helpful for analysts to consider additional information to select optimally best matched service to the requirements.
\end{abstract}

Index Terms - user feedback analysis, services, requirements

\section{INTRODUCTION}

Service Oriented software development has offered a way for the developers to reuse existing software services to build systems while reducing the time and cost of development. In the Service Oriented Requirements Engineering (SORE) there is an additional task of alignment of services to requirements, to find the best matched service for the requirements of all concerned stakeholders while making a trade-off between cost, functional and non-functional requirements. Alignment of requirements to services is considered to be the most challenging and problematic task in SORE due to the following reasons [9]:

Lack of Context: The services are designed and developed free of context to target the needs of larger group of customers. It is sometimes challenging to align the context free services, to the requirements of a project, which has a specific contextual details attach to it.

Missing Information: The specifications published by the service providers may not provide all the information that is required by the analysts to make the decision for its selection.

Mismatch in the Level of Abstraction: Service specifications may not be providing the information and the details about its functionalities at the same level of abstraction as the requirements.

Mismatch in the Level of Granularity: Service granularity refers to the range of functionalities offered by the service.
Services can either be fine-grained (focused, limited functionality) or coarse-grained (broader functionality). Fine granularity offers more flexibility and reusability in customizing the system but also results in increasing the effort and cost of integration. A coarse-grained service is typically expected to carry out more functions but would also exchange more messages and data. This reduces the reusability as the functionality might be too overloaded for the actual need of most consumers.

Paradox of choice: Over the past few years the number of online services has increased exponentially. The analysts now face the problem of 'over choice' while selecting a service form a huge number of available options that offer more or less similar functionalities. With situations where hundreds of alternative choices are available, the decision making for service selection becomes very difficult.

Service selection is an important decision making process and effects the outcome of the resulting system. There are various solutions proposed for selection of services (e.g. model based transformation [1], iterative process [2], granularity analysis [3-5]) but they focus mainly on the technical side of the problem of alignment and do not provide satisfactory and generalizable solutions to the challenges of alignment described above. Technical solutions are only one part of the multifaceted problem. Technology plays an important role in providing solutions but it is equally important to take into account the acceptance of a solution by the people who will ultimately use the resulting system. Without customers' satisfaction a system will be considered as a failure no matter how much sound the use of technology was in developing it.

This paper presents the preliminary results of an empirical study carried out to examine the impact of user feedback on the outcomes of alignment of services to the requirements. The study utilizes our previously proposed method of alignment presented in [20]. Our aim for the study was to refine the proposed method based on the results and perform the preliminary validation of working of the method. The construction of the proposed method was grounded in the findings and systematic analysis of the literature [6,7, 22, 23], a quantitative study (online survey) [8], and qualitative study (interviews with practitioners) $[9,10]$. 


\section{USER FEEDBACK ANALYSIS}

The benefits of involving users in various stages of software development have been studied extensively from various perspectives for more than four decades [7]. In Information System literature, user involvement is intuitively and axiomatically accepted to play a positive key role in users' satisfaction and ultimately leading to the system success. Users can play consultative, informative, participative roles during software development [7]. In consultative and informative role, the users are required to provide the necessary information that can impact the decision making processes of the system development. In service oriented paradigm, user involvement is needed in order to provide personalized systems that can be customized for individual user needs [15]. Although users may not be known and available at the time of development but there is extensive amount of feedback, reviews and comments available by the users of the service on social media, forums and blogs by the previous users of the service. For service oriented development, the users can be approached through their 'voice' from online resources and their feedback can be analyzed to elicit the require information. In recent years, there has been a substantial body of research for proposing methods, tools and techniques on collecting and analyzing online users' feedback, comments and review for extracting useful information [11-17] (e.g. data mining, information retrieval, crowd sourcing, parsing, natural language processing (NLP), sentiment analysis). The user comments and feedback has been a major source of evolution in product line release in case of mobile apps [13, 18, 19]. Various Sentiment analysis tools [21] are available that are designed using NLP, Computational Linguistics, Text mining and analytics capabilities for calculating quantitative values of various users' attitude and behaviour towards a particular phenomenon or a product. Sentiment analysis (also known as opinion mining) helps in monitoring the attitude and behaviour of the users who write about the entity (in our case, a service) on the online social media.

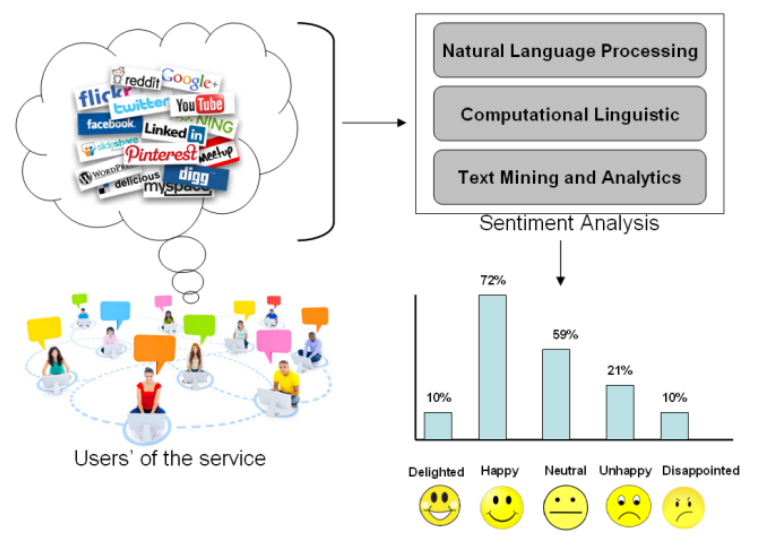

Figure 1. Working of a Sentiment Analysis Tool

User feedback and sentiment analysis offers the decisionmakers the ability to monitor the voice of people in form of their feedback, reviews and comments in order to make more informed decisions. Various studies has demonstrated the benefits of user feedback analysis in different RE areas e.g. Requirements Elicitation [12-14]. However the impact of user feedback has not yet been explored in the alignment process of service oriented development.

\section{Alignment Method FOR Service SELECtion}

Previously we have proposed a method of alignment that provides mechanism for involving users of the services through their feedback [20], Figure 2 represents the overall process where along with requirements and service specifications, the user feedback is analyzed to select the optimally best aligned service among available options. This method is proposed to assist the decision making for service selection thus overcoming the challenges of alignment described above. Our method utilises Multi Criteria Decision Analysis (MCDA) techniques, Requirements Prioritization and Sentiment Analysis for service selection decision making [20].

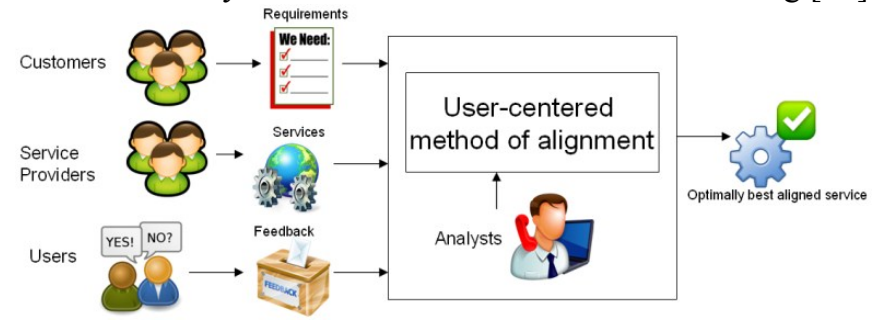

Figure 2. Proposed Method for Alignment [20]

In our proposed method, requirements elicitation results in an initial set of requirements. Using the elicited requirement, analyst would search for available related services from service repositories. Available services can be represented by $\boldsymbol{S}=\left\{\boldsymbol{S}_{1}, \boldsymbol{S}_{2}, \boldsymbol{S}_{3} \ldots \boldsymbol{S}_{X}\right\}$ where $\boldsymbol{X}$ is number of services found.. The resulting service specifications can help in refining or improving the requirements if other innovative features are discovered. The analyst converts the requirements into checklist in consultation with customers and development team. The requirements are prioritized by ranking or assigning weights to the checks based on customer preferences. The set of checks is represented by $\boldsymbol{C}=\left\{\boldsymbol{C}_{1}, \boldsymbol{C}_{2}, \boldsymbol{C}_{3} \ldots \boldsymbol{C}_{\boldsymbol{Y}}\right\}$ and the weights against these checks is represented by $W=\left\{W_{1}, W_{2}\right.$, $\left.W_{3} \ldots W_{Y}\right\}$ where $\boldsymbol{Y}$ is the number of checks in the checklist. The analyst evaluates service specifications to score them (from 0 to 1 ) to find the level of granularity of the service. If there is some missing information then the user feedback is either retrieved from online sources or elicited to get the required information with appropriate techniques (e.g. feature extraction, information retrieval, survey questionnaire). Once all the information is sufficiently complete the checklist scores are generated for all $\boldsymbol{X}$ candidate services. For a service $\boldsymbol{S}_{\boldsymbol{i}}$ from the set of services $\boldsymbol{S}$ the score is represented by Score $_{i}$ which is calculated by adding all the scores achieved to the $\boldsymbol{Y}$ number of checks in set $\mathbf{C}$ multiplied by their respective weights. The service(s) with highest score are those with the highest coverage of stated requirements in the checklist.

$$
\text { Score }_{i}=\sum_{i=1}^{Y}\left(\left(C_{i} * W_{i}\right)\right)
$$

The next step is to analyse the user feedback against the reduced list of selected services after scoring using appropriate 
MCDA method. The user feedback, comments and reviews are collected from the online resources with the help of web search engines and crawlers. They are then analysed with the help of sentiment analysis tools. Among all of the selected services, the service that has the highest positive sentiment score is considered to be the optimally best-aligned service.

\section{THE EMPIRICAL STUDY}

We carried out an empirical study to instantiate the execution of our method in practice. The objectives for this study were twofold: (1) refinement and improvement of the method, and (2) initial validation of the idea of involving user feedback in the service selection process. The study involved the selection of an SMS gateway service for an existing website that belongs to a gym. This service enables websites to send and receive text or multimedia messages with simple invocation of the remote service API while hiding all the underlying technical and infrastructure details. The gym required the SMS facility in their online system to contact its registered members considering that it is faster to approach them through SMS rather than email. There are currently 241 registered members and the number varies from 200 to 350 in one month. All of the members are located in Sydney. The gym needs to send single, group, or broadcast messages only in Australia to its members for registration and different notification purposes. The frequency of sending one SMS to a member is higher than sending bulk messages to all the members therefore the gym is looking for a service that provides cheaper rates per SMS. In addition to the cost and the basic functional requirements, the reliability and timely communication are the top priorities especially when sending activation code to mobile phones for signing up as a new member.

\section{A. Execution of the Method}

Online searches resulted in 92 eligible options providing the SMS gateway services. From the customer requirements 28 checks were created for evaluating these services. The requirements became further refined and became more complete later when we were analysing service specification with better understanding of the functionalities of the available services. Table I shows the checks prioritized with the weights assigned to them by the customers and the development team. This created a complex and challenging scenario for decision making as many of the services offered more or less the same functionality within the same price range.

As a first step, we evaluated all 92 services to score them against the requirements checklist as given in Table I. The scores were given as yes $=1, \mathrm{no}=0$ and partial $=0.5$. The scores are calculated based on the information provided in the service specifications on the websites and API documents. For the checks where the information was not given by service provider (in 70\% cases this happened for checks 6, 7 and 23), we searched online forums and blogs where reviews were available about the services and analysed the comments with keyword searching to find out the required information. Though we were only able to retrieve the missing information for 15 services, but considering the fact that we performed the task manually, using sophisticated information retrieval tools would help significantly.

TABLE I. PRIORITIZED CHECKLIST OF REQUIREMENTS

\begin{tabular}{|l|l|l|}
\hline $\boldsymbol{C}_{\boldsymbol{i}}$ & Check Description & $\boldsymbol{W}_{\boldsymbol{i}}$ \\
\hline 1 & Service supports outgoing text messages in Australia & 13 \\
\hline 2 & Service should not have any hardware or SIM requirements & 13 \\
\hline 3 & Service should be highly reliable with 99.9\% message delivery & 10 \\
\hline 4 & Cost of service should not be more than 0.1 AUD per SMS & 10 \\
\hline 5 & There should be no set up cost & 10 \\
\hline 6 & There should be no hidden charges & 10 \\
\hline 7 & All charges (tax) should be included in credit for SMS & 10 \\
\hline 8 & There should be no expiry on SMS credit & 10 \\
\hline 9 & Payment should be done by credit card or Bpay & 10 \\
\hline 10 & Service API should provide SOAP and HTTP support & 10 \\
\hline 11 & Service API should support PHP script & 10 \\
\hline 12 & No customization should be required in service & 10 \\
\hline 13 & Service should be compatible with any web browser & 10 \\
\hline 14 & Automatic connection should be established with every API call & 10 \\
\hline 15 & Service should provide simple authentication on every API call & 10 \\
\hline 16 & Service supports text messages in English & 9 \\
\hline 17 & Service supports basic 160 characters per message & 9 \\
\hline 18 & Service supports sending a notification text message to one member & 9 \\
\hline 19 & Service supports sending a validation code message to one member & 9 \\
\hline 20 & Service supports sending notification message to multiple members & 9 \\
\hline 21 & Service supports sending notification message to all the members & 9 \\
\hline 22 & Service supports text composed by the administration & 9 \\
\hline 23 & SMS transmission delay should be between 3 to 7 seconds & 9 \\
\hline 24 & Service should offer contacts management & 9 \\
\hline 25 & Service supports retrieval of send messages record & 7 \\
\hline 26 & Service shows message delivery message & 7 \\
\hline 27 & Service shows message delivery failure notification & 7 \\
\hline 28 & Service should provide schedule message delivery & 5 \\
\hline
\end{tabular}

Once all the scores were calculated the next step was to get the quantitative values for the user feedback, reviews and comments from online sources with the help of sentiment analysis tools. We selected a free online tool socialmention.com for this purpose. It helps in tracking any mention for the identified keywords in video, blogs, events, news, bookmarks, hashtags and even audio files. It categorizes the results into three types of sentiments i.e. positive, neutral and negative. It also gives values for "Passion" which is a measure of the likelihood that people who are talking about the product or service will do so repeatedly, and for "Reach" which is the measure of the range of influence of the product or service.

\section{B. Results}

Out of 92 there were 16 services whose overall score was above 200 . Due to the lack of space we only describe results for the top five services which are in close competition shown in Table II. According to these results service 19 can be considered as the best aligned service. But service 76, 68, 1 and 71 are also close competitors.

TABLE II. SERVICES WITH SCORE ABOVE 200

\begin{tabular}{|l|l|l|}
\hline \multicolumn{1}{|c|}{ Service $_{i}$} & Link & Score $_{i}$ \\
\hline Service 19 & Intel Tech http://inteltech.com.au & 259 \\
\hline Service 1 & Clickatell $\underline{\text { http://www.clickatell.com.au }}$ & 258 \\
\hline Service 68 & Skebby http://www.skebby.com & 258 \\
\hline Service 76 & Via SMS http://viasms.com.au/ & 258 \\
\hline Service 71 & Direct SMS http://www.directsms.com.au/ & 257 \\
\hline
\end{tabular}

The sentiment analysis results as shown in Table III were generated on $30^{\text {th }}$ May 2014. After analysing the data retrieved form the sentiment analysis tools, service 1 (Clickatell) appeared as a way more popular and has significantly more 
positive results for its mention on the social media when compared to the service 19 (Intel Tech). Furthermore, services 76 and 68 while have achieved the same score but do not show better results in the sentiment analysis of table III. Table II shows that the difference between the overall scores of services 19 and 1 is only by one point. Therefore, referring to the paradox of choice explained above, we suggest that the analyst can make a more informed decision on which one to select (between services 1 and 19) based on the information retrieved by the end user feedback.

TABLE III. RESULTS FROM SOCIALMENTION.COM

\begin{tabular}{|l|l|l|l|l|l|}
\hline \multirow{2}{*}{ Service } & \multicolumn{5}{|c|}{ Socialmention.com } \\
\cline { 4 - 6 } & \multirow{2}{*}{ Passion } & \multirow{2}{*}{ Reach } & \multicolumn{3}{|c|}{ Sentiment Frequency } \\
\cline { 4 - 6 } & & & Positive & Neutral & Negative \\
\hline Service 19 & $0 \%$ & $4 \%$ & 2 & 54 & 2 \\
\hline Service 1 & $28 \%$ & $25 \%$ & 76 & 96 & 0 \\
\hline Service 68 & $12 \%$ & $24 \%$ & 5 & 86 & 0 \\
\hline Service 76 & $23 \%$ & $26 \%$ & 7 & 94 & 0 \\
\hline Service 71 & $82 \%$ & $3 \%$ & 3 & 21 & 0 \\
\hline
\end{tabular}

Our study has demonstrated that evaluating a service with the information advertised by the service provider in their specification may not always lead to an optimal decision for service selection. The social media presents a new opportunity for monitoring trends and sentiments of users towards a particular service among a large pool of options. Users who have the past experience of using a service can provide valuable information that can be helpful in decision making process. We should point out that in this study we have only used the quantitative scores provided by the sentiment analysis tools. There was a significant amount of textual reviews retrieved which can be analysed qualitatively for contextual information retrieval regarding the service usage by previous users. The result of this qualitative analysis in combination with the quantitative scores would then provide a more complete picture of each service for optimal selection.

\section{CONCLUSION AND FUTURE WORK}

The study reported in this paper was carried out with two specific aims: (1) refinement and improvement of our proposed method, and (2) preliminary validation of the idea of involving user feedback in the service selection process. The novelty of our method is to involve the voice of users with the help of sentiment analysis in the service selection process as well as utilising MCDA in the decision analysis. We believe that we have achieved both aims. Firstly, the conduct and the results of this study have helped improve our method in refining the order and the details of the steps required for granularity analysis. Secondly, this study has further informed our long term plan of full evaluation of our method. We now plan to conduct a controlled experiment with practitioners on real industry cases to fully assess the robustness and reliability of this method supported by an automated tool that we are currently designing. This study was helpful in refining the proposed method by showing the details of all the steps required to be carried out in practice. This was necessary for the design and development of our supporting tool to reduce the information overload and assist the analysts to simulate the results for evaluating different options.

\section{REFERENCES}

[1] A. Gehlert, N. Bramsiepe, and K. Pohl. Goal-driven alignment of services and business requirements. in IEEE Service-Oriented Computing: Consequences for Engineering Requirements, 2008. SOCCER'08.

[2] K. Zachos, N. Maiden, and R. Howells-Morris, Discovering web services to improve requirements specifications: does it help?, in Requirements Engineering Foundation for Software Quality 2008, p. 168-182.

[3] C. Steghuis, "Service granularity in SOA projects: a trade-off Analysis", Master's thesis, University of Twente, 2006.

[4] Heinrich, B., M. Henneberger, A. Krammer, F. Lautenbacher, Granularity of services-an economic analysis. Business \& Information Systems Engineering, 2011.3(6): p. 345-358.

[5] N. Kulkarni, V. Dwivedi. The role of service granularity in a successful SOA realization a case study. in IEEE Congress on Services, 2008.

[6] M Bano, N. Ikram. Issues and challenges of requirement engineering in service oriented software development. in IEEE Fifth International Conference on Software Engineering Advances (ICSEA), 2010.

[7] M. Bano, D. Zowghi. User involvement in software development and system success: a systematic literature review. in Proceedings of the 17th International Conference on Evaluation and Assessment in Software Engineering. 2013. ACM.

[8] M. Bano, N. Ikram. KM-SORE: knowledge management for service oriented requirements engineering. in The Sixth International Conference on Software Engineering Advances (ICSEA). 2011.

[9] M. Bano, D. Zowghi, N. Ikram, M. Niazi., What makes service oriented requirements engineering challenging? A qualitative study. IET Software 2013.

[10] M. Bano Sahibzada, and D. Zowghi. Service Oriented Requirements Engineering: Practitioner's Perspective. in Service-Oriented Computing-ICSOC 2012 Workshops. 2013. Springer.

[11] J. Hao, S. Li, and Z. Chen, Extracting service aspects from web reviews, in Web Information Systems and Mining. 2010, Springer. p. 320-327.

[12] N. Seyff, F. Graf, and N. Maiden. Using mobile re tools to give end-users their own voice. in 18th IEEE International Requirements Engineering Conference (RE), 2010.

[13] L. V. Galvis Carreño, K. Winbladh. Analysis of user comments: an approach for software requirements evolution. in 2013 IEEE International Conference on Software Engineering (ICSE) pp. 582-591.

[14] N. Seyff, F. Graf, and N. Maiden. End-user requirements blogging with iRequire. in Proceedings of the 32nd ACM/IEEE International Conference on Software Engineering (ICSE) 2010.

[15] D. Pagano, B. Bruegge. User involvement in software evolution practice: a case study. in Proceedings of the IEEE International Conference on Software Engineering (ICSE) 2013.

[16] D. Pagano, W. Maalej. User feedback in the appstore: An empirical study. in International Requirements Engineering Conference 2013.

[17] M. Harman, Y. Jia, and Y. Zhang. App store mining and analysis: MSR for app stores. in 9th IEEE Working Conference on Mining Software Repositories (MSR), 2012.

[18] B. Fu, J. Lin, L. Li, C. Faloutsos, J. Hong, N. Sadeh. Why people hate your app: making sense of user feedback in a mobile app store. in Proceedings of the 19th ACM SIGKDD international conference on Knowledge discovery and data mining. 2013

[19] M. Chen, X. Liu. Predicting popularity of online distributed applications: iTunes app store case analysis. in 2011 iConference. ACM.

[20] M. Bano, N. Ikram, Addressing the Challenges of Alignment of Requirements and Services: A Vision for User-Centered Method, in Requirements Engineering. 2014, Springer. p. 83-89.

[21] B. Pang, L. Lee, Opinion mining and sentiment analysis. Foundations and trends in information retrieval, 2008. 2(1-2): p. 1-135.

[22] M. Bano, D. Zowghi, A Systematic Review on the Relationship between User Involvement and System Success. Information and Software Technology, 2014 doi: http://dx.doi.org/10.1016/j.infsof.2014.06.011

[23] M. Bano, D. Zowghi 'Users' involvement in requirements engineering and system success', in IEEE Third International Workshop on Empirical Requirements Engineering (EmpiRE) at RE Conference, 2013, pp. 24-31 